\title{
Melanocortin 4 receptors switch reward to aversion
}

\author{
Alexandra G. DiFeliceantonio ${ }^{1,2}$ and Paul J. Kenny' \\ 'Department of Neuroscience, Icahn School of Medicine at Mount Sinai, New York, New York, USA. ²Department of Psychiatry, Yale School of Medicine, New Haven, Connecticut, USA.
}

\begin{abstract}
The ability to recognize and avoid noxious stimuli is essential for survival. The factors that determine whether a given stimulus is considered positive or negative are complex and not fully understood. In this issue of the JCI, Klawonn and colleagues demonstrate that melanocortin 4 receptor (MC4R) signaling is critical for proper responses to negative stimuli. Mice lacking MC4R were shown to have a surprising preference for aversive stimuli compared with WT animals. Moreover, the authors provide evidence that avoidance behaviors are mediated by hypothalamic POMC neurons signaling to striatal dopamine D1 receptor-expressing medium spiny neurons. Together, these results provide important insight into the regulation of responses to aversive stimuli.
\end{abstract}

\section{To avoid or to approach?}

Successful navigation of agiven environment requires necessary avoidance of noxious stimuli and the recognition and approach of positive stimuli. Complex computations are involved in determining whether novel environmental stimuli are positive or averse (assigning valence) and in coordinating the appropriate escape or approach behaviors; therefore, a better understanding of how stimuli are deemed attractive or noxious is a subject of much interest. An important question in this regard is whether single brain systems respond to rewarding and aversive stimuli along a continuum or separate systems discretely encode rewarding and aversive stimuli, with interactions between these opposing systems influencing the selection of appropriate behavioral strategies. In this issue, Klawonn and colleagues (1) provide evidence that supports a two-system mechanism for avoiding noxious stimuli. Surprisingly, this study suggests that deficits in brain-aversion systems regulated by the melanocortin 4 receptor (MC4R) can reverse the valence of otherwise noxious stimuli such that animals will seek out and approach these threatening stimuli as if they were rewarding. Targeting the MC4R could therefore have important clinical applications for disorders characterized by maladaptive responses to threatening stimuli.

\section{MCR4 regulates response to aversive stimuli}

Two populations of neurons in the arcuate nucleus of the hypothalamus, one that synthesizes the neuropeptides agouti-related peptide (AgRP) and neuropeptide Y (NPY) and one that synthesizes proopiomelanocortin (POMC), are known to exert opposite controls over the foraging and consumption of food. These neurons project to the paraventricular nucleus of the hypothalamus, where melanocortin released from POMC neurons activates MC4R to inhibit feeding behaviors and AgRP acts as an inverse agonist at the MC4R to stimulate feeding. POMC and AgRP neurons project to other brain regions, including the striatum, but less is known about their actions outside the hypothalamus. Klawonn and colleagues (1) investigated the possibility that MC4R expression in the striatum can regulate the approach and avoidance of non-food-relevant environmental stimuli.

First, Klawonn et al. (1) used mutant mice in which the MC4R-encoding gene had

Related Article: p. 3160

Conflict of interest: PJK is cofounder of Eolas Therapeutics Inc. and is a scientific advisor to Takeda Pharmaceuticals USA. Reference information: J Clin Invest. 2018;128(7):2757-2759. https://doi.org/10.1172/JCI121653.

been genetically deleted. Unlike WT animals, $M c 4 r$-KO mice did not avoid an environment that had been repeatedly paired with a noxious stimulus. Instead, $\mathrm{Mc} 4 r-\mathrm{KO}$ mice spent more time in the noxious environments, while WT mice spent more time exploring an environment in which they had previously encountered a rewarding stimulus. This surprising effect was seen regardless of the type of noxious stimulus, including those that induced sickness, nausea, or pain. Moreover, WT mice showed a preference for noxious stimuli when treated with an MC4R antagonist. Other, nonapproach-related behavioral responses to noxious stimuli, such as reduced appetite, were unaffected in $M c 4 r$-KO mice. Similarly, MC4R-deficient mice responded normally to positive stimuli, such as palatable food or cocaine. Together, these unusual observations suggest that, in the absence of MC4R signaling, the motivational valence of otherwise noxious stimuli flips; therefore, instead of avoiding threatening stimuli, MC4Rdeficient mice view these stimuli to be as attractive as appetizing food.

The process of associating a rewarding stimulus with a particular environment is thought to be mediated by striatal dopamine transmission. Klawonn and colleagues, therefore, explored the possibility that, in the absence of MC4R, noxious stimuli enhance dopamine transmission in a manner similar to that of rewarding stimuli, thereby triggering maladaptive approach behavior. LPS, which induces sickness and malaise in rodents, reduced striatal dopamine levels in WT mice, but elevated dopamine levels in $M c 4 r$-KO mice, as measured indirectly by using PET to detect dopamine-mediated displacement of $\left[{ }^{11} \mathrm{C}\right]$-raclopride binding in the striatum. Moreover, chemogenetic inhibition of dopamine neurons in the ventral tegmental area (VTA) or pharmacological antagonism of dopamine D1 receptors (D1Rs) blocked the preference of $M c 4 r-K O$ mice for environments paired with noxious stimuli. These findings suggest that perturbations in dopamine transmission in 


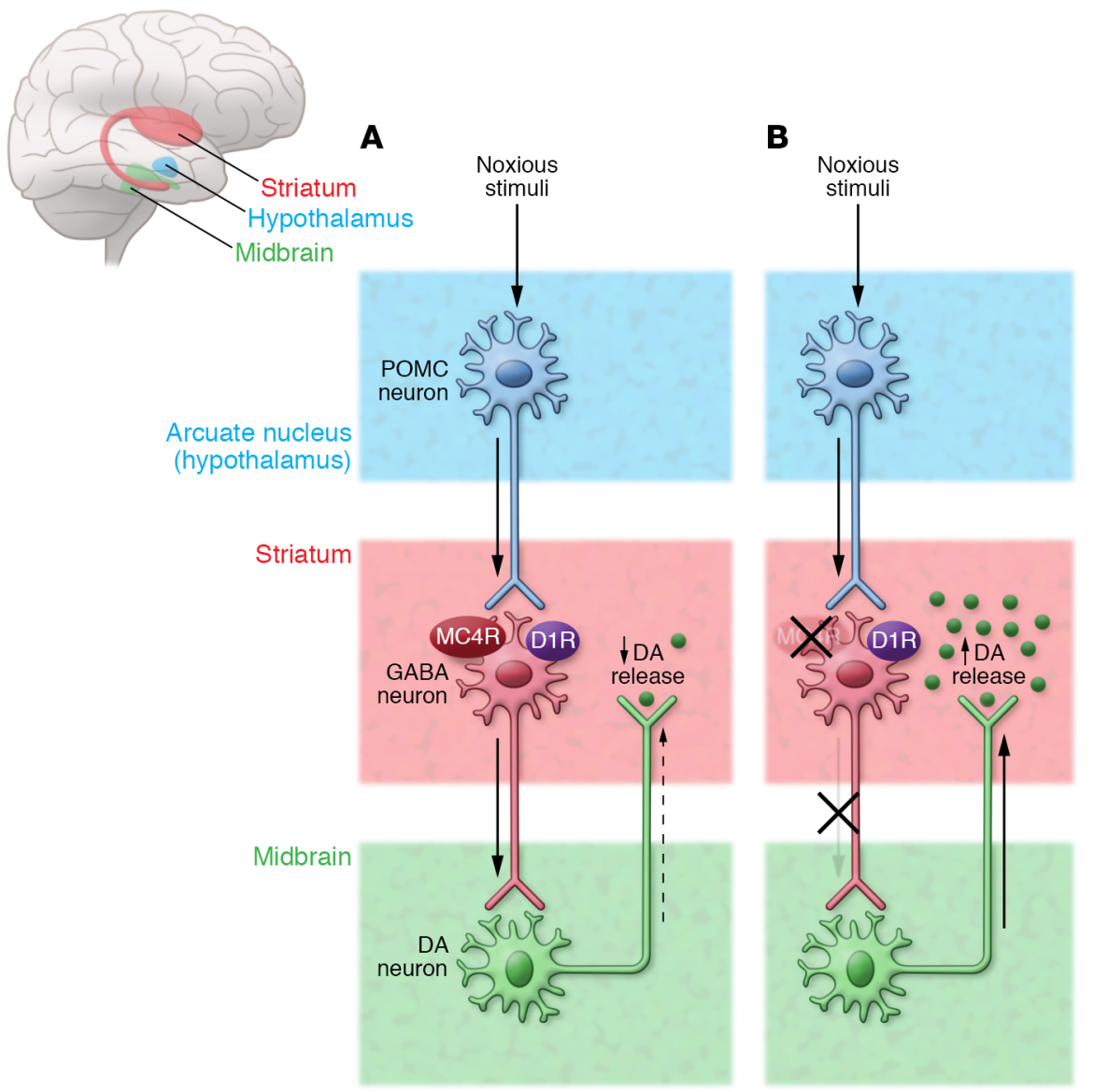

Figure 1. POMC neurons act through MC4R receptors in striatum to control avoidance behavior. POMC-producing neurons in arcuate nucleus are activated by noxious stimuli. These neurons project to the striatum, where they activate D1R-expressing MSNs through MC4Rs. These D1R-expressing cells are likely those that project to the midbrain to inhibit dopamine neurons. In the absence of MC4R signaling, POMC neurons do not activate D1R-expressing MSNs in striatum. This results in disinhibited dopamine transmission and the formation of maladaptive positive associations between noxious stimuli and the environments in which they are encountered. DA, dopamine.

Mc4r-KO mice underlie the preference for noxious stimuli. PET imaging is a rather blunt tool for investigating the dynamics of dopamine transmission, and more precise techniques, such as in vivo microdialysis or fast-scan cyclic voltammetry, will provide better understanding of dopaminesignaling disturbances in $\mathrm{Mc} 4 \mathrm{r}$-KO mice. However, an advantage of PET is that it provides a striatum-wide view of dopamine transmission. Interestingly, PET imaging revealed that only a portion of the dorsal striatum, rather than ventral domains of the striatum that are typically implicated in reward responses, showed altered dopamine transmission in the $M c 4 r$ KO mice. This potentially fascinating subdivision of the dorsal striatum, which appears to attribute valence to rewarding and aversive stimuli, fits with an emerging body of literature that implicates the dorsal striatum
Mc4r-KO mice to noxious stimuli and because dopamine transmission may regulate behavioral responses to aversive stimuli (4), Klawonn and colleagues evaluated the role for D1R-MSNs in the behavior of $M c 4 r$-KO mice by using an elegant genetic approach to selectively reexpress the MC4R only in D1R-MSNs of Mc4r-KO mice. D1R-MSN-specific reexpression of MCR4 restored normal avoidance of noxious stimuli, supporting MC4R signaling in D1R-MSNs as a regulator of the valence of noxious and rewarding stimuli.

Finally, Klawonn and colleagues examined whether POMC neurons originating in the arcuate nucleus are responsible for activating MC4Rs in the striatum to control approach and avoidance behaviors. Optogenetic stimulation of the terminals of POMC neurons in the striatum increased the firing rate of striatal MSNs. Moreover, chemogenetic stimulation of POMC neurons that project to the striatum increased avoidance-related behaviors. These observations confirm that hypothalamic POMC neurons act on striatal MSNs, presumably the same population of D1R-MSNs that express MC4Rs, to regulate avoidance behaviors.

\section{Concluding remarks}

Together, the findings of Klawonn and colleagues provide unexpected yet compelling evidence that inputs from hypothalamic POMC neurons to striatal D1RMSNs control avoidance of a wide range of aversive and potentially threatening stimuli. These are important findings because they identify a brain circuit that is involved in signaling aversion. Strikingly, disruption of this circuit does not render animals ambivalent to aversive stimuli. Instead, it flips the valence so that threatening stimuli become attractive. Such dopaminedependent striatal switches have been described previously in the shell region of the nucleus accumbens $(5,6)$. The current findings of Klawonn et al. extend these findings to the dorsal striatum and suggest that inputs from the hypothalamus play an important role in determining how the striatum codes the valence of environmental stimuli. Many unanswered questions remain, however. For example, does this MC4R-regulated avoidance circuit act in isolation or does it communi- 
cate with other brain regions involved in avoidance behaviors, such as the habenula? In addition, how precisely does blockade of MC4R signaling result in increased dopamine transmission in response to noxious stimuli? One possibility is that MC4Rregulated D1R-MSNs are those that provide inhibitory input to midbrain dopamine such that loss of MC4R signaling disinhibits dopamine neurons at times when they should be silenced (see Figure 1). Further experiments will be required to answer these important questions. Nevertheless, the striking effects of MC4R inhibition on avoidance behaviors identifies these receptors as potentially important targets for the development of interven- tions for disorders characterized by pathologically enhanced avoidance behavior and aversion.

\section{Acknowledgments}

This work was supported by a grant from NIDA (DA025983) to PJK.

Address correspondence to: Paul J. Kenny, Department of Neuroscience, Icahn School of Medicine at Mount Sinai, 1470 Madison Avenue, New York, New York 10029, USA. Phone: 212.824.8970; Email: paul.kenny@ mssm.edu.

\footnotetext{
1. Klawonn AM, et al. Motivational valence is determined by striatal melanocortin 4 receptors. JClin Invest. 2018;128(7):3160-3170.
}

2. London TD, et al. Coord;inated ramping of dorsal striatal pathways preceding food approach and consumption. J Neurosci. 2018;38(14):3547-3558.

3. Cole S, Stone AD, Petrovich GD. The dorsomedial striatum mediates Pavlovian appetitive conditioning and food consumption. Behav Neurosci. 2017;131(6):447-453.

4. Badrinarayan A, et al. Aversive stimuli differentially modulate real-time dopamine transmission dynamics within the nucleus accumbens core and shell. J Neurosci. 2012;32(45):15779-15790.

5. Richard JM, Berridge KC. Metabotropic glutamate receptor blockade in nucleus accumbens shell shifts affective valence towards fear and disgust. Eur J Neurosci. 2011;33(4):736-747.

6. Richard JM, Berridge KC. Nucleus accumbens dopamine/glutamate interaction switches modes to generate desire versus dread: $\mathrm{D}(1)$ alone for appetitive eating but $\mathrm{D}(1)$ and $\mathrm{D}(2)$ together for fear. J Neurosci. 2011;31(36):12866-12879. 